\title{
Deburring and rounding of sheet metals and functionally relevant edges of parts using laser radiation
}

\author{
Judith Kumstel $^{1, *}$, and Sven Lüken ${ }^{1}$ \\ ${ }^{1}$ Fraunhofer ILT - Institute for Laser Technology, 52074 Aachen, Germany
}

\begin{abstract}
The manufacturing of sheet metal parts or component edges often leads to burrs. Due to different requirements regarding the appearance, haptics, tribology et cetera the edges have to be deburred or rounded in a subsequent process. Especially for complex geometries, automated post-processing has been difficult up to now. Laser remelting is one possible solution. It is fully automated and suitable for most 3D geometries. During laser remelting the laser beam is focused on the surface, thus a thin surface layer up to $100 \mu \mathrm{m}$ is melted. The surface tension in the liquid state is smoothing out the surface and the edge resolidifies rounded. The radius of the rounding is adjustable by the chosen process parameters, especially laser power $\mathrm{P}_{\mathrm{L}}$ and feed speed Vfeed. Furthermore the resulting roughness and the gloss level are controllable via the process parameters. Typical processing speeds are $100 \mathrm{~mm}$ edge length per second. First studies with industry partners showed promising results. Laser remelting of push belts for the torque transmission in CVTs lead to an increase of fatigue strength by up to $200 \%$ which enables a higher torque.
\end{abstract}

Keywords: Deburring, Laser beam machining (LBM), Surface modification

\section{Introduction}

When cutting or separating sheet metal parts, it is often not possible to produce edges free of burrs and defects. Burrs and similar edge defects impair the mechanical properties with negative consequences for processability and loadability (edge formability, fatigue properties). Furthermore they considerably increase the risk of injury and can endanger the functionality of components (e.g. damage to cable insulation, wear).

Although there are many different processes for deburring and edge processing, these have significant shortcomings depending on the task at hand (e.g. contamination of the components and therefore the need for an additional cleaning step, no selective processing and thus damaging of areas that have not to be processed, not suitable for the processing of free-form edges). This sometimes results in high production costs and/or the achievable component quality is limited.

A rather new and very unexplored approach for deburring and edge processing is the laser remelting. Laser remelting of sheet metal is a flexible process and is based on remelting the edge using laser radiation. In the liquid state, the roughness of the edge can flow out due to the surface tension and is smoothed. Burrs and microcracks can also be melted down in this way. In addition, the molten edge solidifies rounded.

A comprehensive investigation of laser deburring of steel materials is shown in [1]. However the investigation is limited to fine burrs with the aim of producing sharpedged, burr-free edges on small components. Although there is already a first industrial implementation of laser deburring in the series production of sheet metal rings for CVT car transmissions, the potential of laser edge processing is only just beginning to be exploited $[2,3]$.
Systematic investigation of the quantitative relationships between the process parameters of laser edge processing and the expected property profiles have not yet been carried out. Also, the application limits of the method have not been identified. This paper examines the influence of the process parameters of laser remelting on the edge rounding.

A similar process based on the same basic principle but developed for other applications and dimensions is the laser rod end melting. Laser rod end melting results in a rounding of the end of cylindrical rods for rods with a maximum diameter of $1 \mathrm{~mm} \mathrm{[4].} \mathrm{After} \mathrm{the} \mathrm{generation} \mathrm{of}$ the spherical geometry at the rod end in the master forming stage, this part can be used as a preform for a micro cold forming operation in a subsequent process stage for the production of metallic micro parts such as screws or springs [5].

\section{Basics of laser remelting}

During laser remelting the laser beam (round or rectangular) is focused on the surface and guided over the surface with the feed speed $\mathrm{v}_{\text {feed }}$, thus a thin surface layer is melted (see fig. 1). The surface tension in the liquid state is smoothing out the surface and the edge resolidifies rounded. No material is removed but rather relocated while molten. For metals, diode-pumped solid-state lasers in CW mode are used. The thickness of the remelted layer is in the range of 50- $400 \mu \mathrm{m}$.

\footnotetext{
* Corresponding author: judith.kumstel@ilt.fraunhofer.de
} 


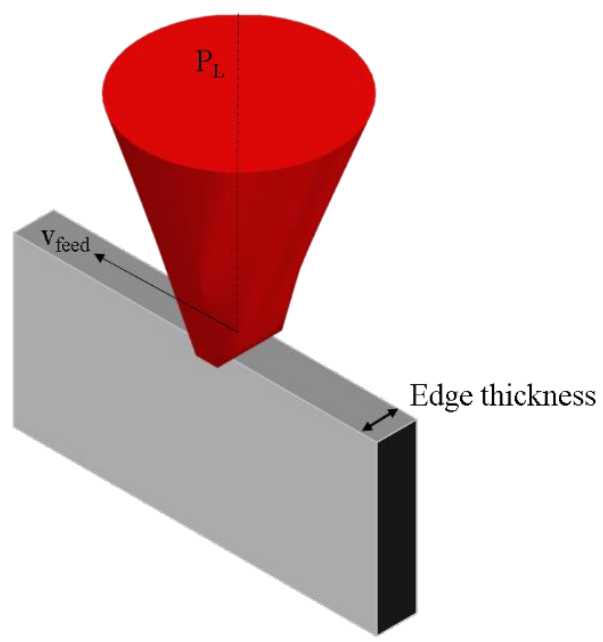

Fig. 1. Process principle for laser edge rounding with round laser beam.

The process parameters (e.g. laser power, feed speed and laser beam shape and size) have to be adapted to the initial roughness and the treated type of material. Furthermore the level of edge rounding can be adjusted by a suitable selection of the process parameters and reaches from simple deburring up to reinforcing the edge (see fig. 2).

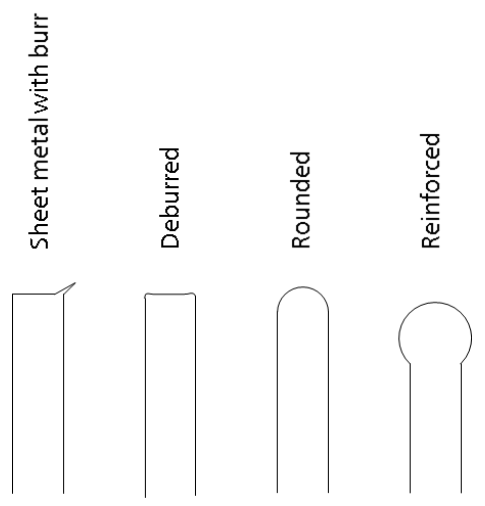

Fig. 2. Possible shapes of the sheet metal edges.

Laser remelting is carried out under inert gas atmosphere to avoid oxidation.

\section{Experimental setup \& approach}

To investigate the influence of the laser beam shape and size on the edge rounding, an experimental setup is developed with which different beam geometries can be realized quickly and cost-effectively (fig. 3). At first, a rectangular top-hat intermediate focus is generated by a micro lens array. The micro lens array rearranges the collimated circular Gaussian beam into a rectangular tophat beam. An adjustable aperture in the following intermediate focus then crops the beam into the desired shape.

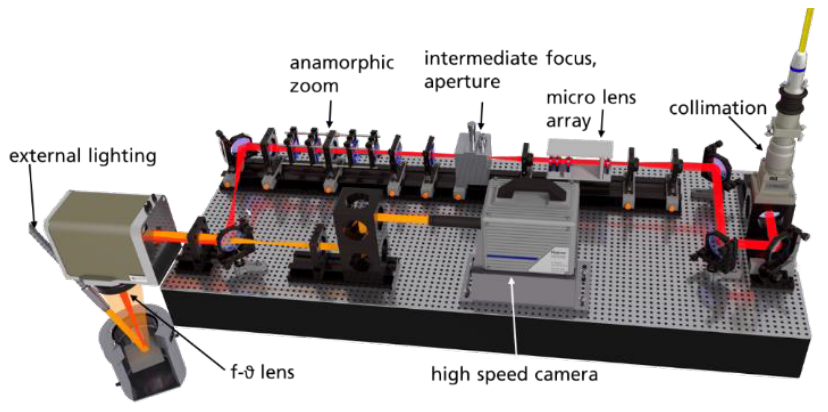

Fig. 3. Experimental Setup

This approach allows a flexible and cost efficient realization of different shaped laser beams, because the manufacturing of different apertures is very quick and cheap. The cut intermediate focus is then projected onto the surface of the sheet metal via a complex optical system, a laser scanner and an $f-\vartheta$ lens. For the variation of the aspect ratio of the beam an anamorphic zoom telescope is integrated. The used laser source is a disc laser from Trumpf with a maximum output laser power of $\mathrm{P}_{\mathrm{L}}=1 \mathrm{~kW}$.

All the investigations are performed on sheet metals of the austenitic stainless steel 1.4301 with an edge thickness of $1 \mathrm{~mm}$ (fig. 4, right).

The approach for the investigation of the cause-andeffect relationship between the shape of the laser beam, the process parameters and the resulting rounding of the sheet metal edge is as follows:

- Sheet metal edges are laser remelted under variation of the shape and size of the laser beam, the laser intensity $I$ and the feed speed $\mathrm{v}_{\text {feed }}$. The varied process parameters are listed in table 1 , the tested shapes of the laser beam are shown in figure 4, left.

Table 1. Varied process parameters.

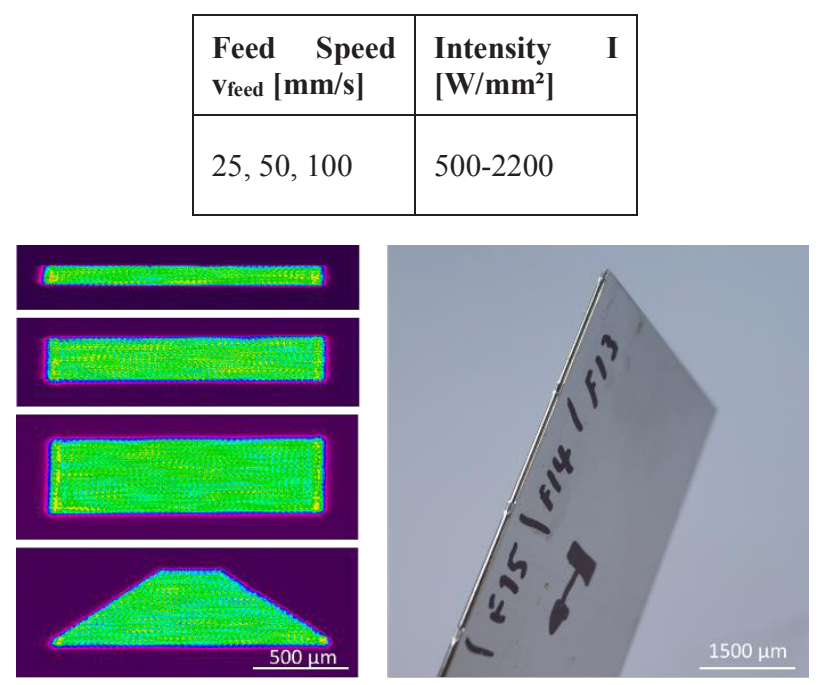

Fig. 4. Left: Overview of the tested laser beam shapes, right: Sample with rounded edges using different process parameters. 
The naming of the shapes is as follows: A line shaped laser beam with a length of $1500 \mu \mathrm{m}$ and a width in scanning direction of $100 \mu \mathrm{m}$ is named "line $1500 \times 100 \mu \mathrm{m}^{2}$ ".

To make statistical statements, each parameter combination is tested three times.

- The laser remelted sheet metal edges are analyzed concerning the achieved rounding with a white light interferometer. By shooting at different heights, the rounded edge can be precisely displayed with the white light interferometer (transversal resolution: $0.1 \mathrm{~nm}$, lateral resolution: $\sim 100 \mathrm{~nm}$ ), so that the radius can be determined.

- To get an impression of the surface quality after laser remelting, light microscopy photos of the edges are taken (fig. 5, right).

- To measure the depth of remelting, cross sections are prepared (fig. 5, left).
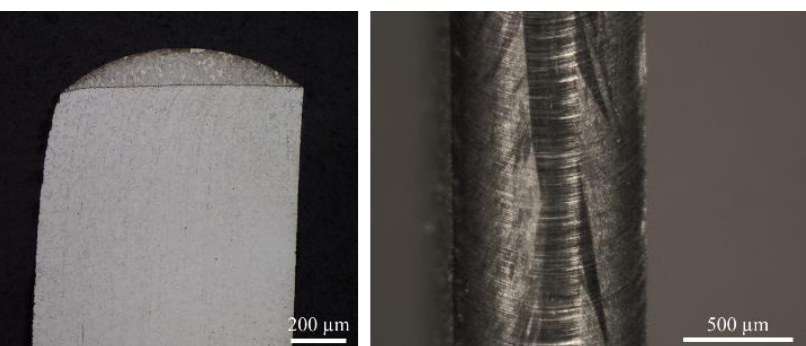

Fig. 5. Left: Cross section through a laser remelted edge, right: light microscopy of the top of the remelted edge (process parameters: line $1500 \times 100 \mu \mathrm{m}^{2}, \quad \mathrm{~V}_{\text {feed }}=25 \mathrm{~mm} / \mathrm{s}$, $\left.\mathrm{I}=2000 \mathrm{~W} / \mathrm{mm}^{2}\right)$.

\section{Results}

In a first step the influence of feed speed $v_{\text {feed }}$ and intensity I on the resulting radius of the remelted edges are analysed for the line shaped laser beam of $1500 \times 100 \mu \mathrm{m}^{2}$ (fig. 6).

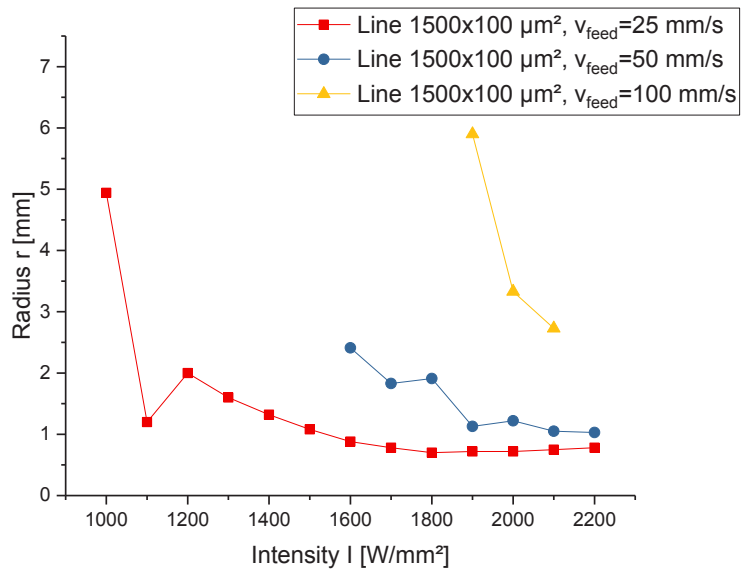

Fig. 6. Average radius $r$ of the laser remelted edge at different feed speeds $v_{\text {feed }}$ and intensities I for the line shaped laser beam $1500 \times 100 \mu \mathrm{m}^{2}$.

The reproducibility of the results is very high, the standard deviation is between 0.01 and $0.04 \mathrm{~mm}$. Due to their small sizes the error bars in the graphs are not visible. The achievable radius increases for a faster scanning. A reason for this behavior could be the smaller remelting depth for faster feed speeds and same intensities, because the shape of the molten pool differs depending on the feed speed (fig. 7).

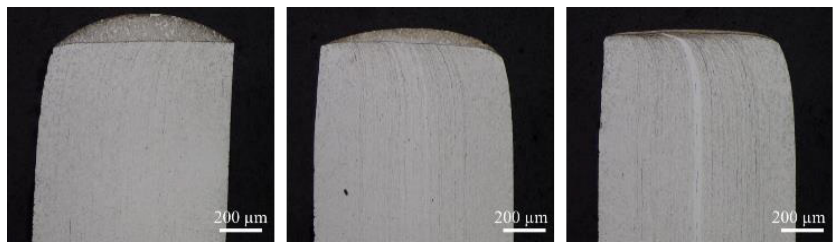

Fig. 7. Cross section through laser remelted edges with different feed speeds $v_{\text {feed }}=25 \mathrm{~mm} / \mathrm{s}$ (left), $v_{\text {feed }}=50 \mathrm{~mm} / \mathrm{s}$ (middle), $\mathrm{Vfeed}_{\mathrm{f}}=100 \mathrm{~mm} / \mathrm{s}$ (right) (process parameters: line 1500x100 $\mu \mathrm{m}$, $\left.\mathrm{I}=2000 \mathrm{~W} / \mathrm{mm}^{2}\right)$.

For the considered laser beam geometry and feed speed, it is not possible to increase the radius further by reducing the intensity, as the process becomes unstable and the sheet metal edge is no longer remelted completely.

For $\mathrm{v}_{\text {feed }}=25 \mathrm{~mm} / \mathrm{s}$ the influence of the line width on the rounding of the laser remelted edge has been analysed (fig. 8). For this the length of the line shaped laser beam has been kept constant at $1500 \mu \mathrm{m}$, only the width in scanning direction has been varied from $100 \mu \mathrm{m}$ to 500 $\mu \mathrm{m}$. 


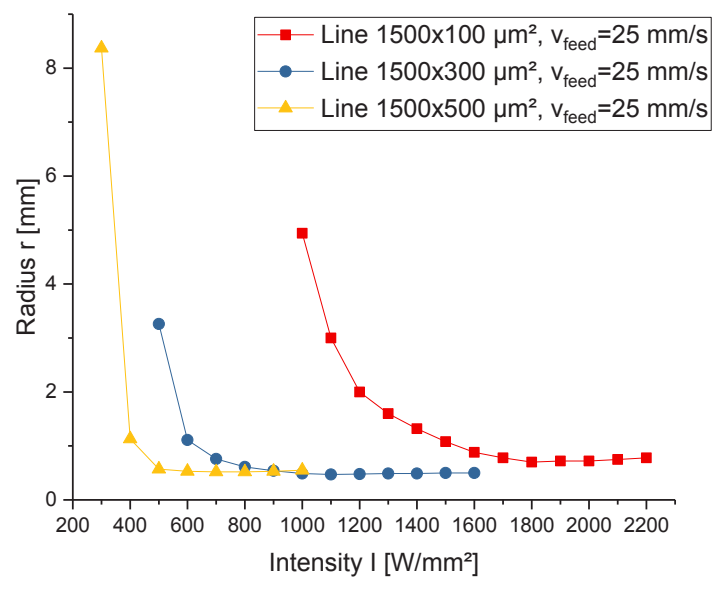

Fig. 8. Average radius $r$ of the laser remelted edge for different line widths and intensities I at a (constant) feed speed of $\mathrm{V}$ feed $=25 \mathrm{~mm} / \mathrm{s}$.

It seems that the minimal achievable radius is independent of the laser beam shape. For higher intensities each graph becomes saturated with $\mathrm{r} \approx 0.55 \mathrm{~mm}$. At a sheet thickness of $1 \mathrm{~mm}$, this corresponds to half the sheet thickness and thus to a spherical bulge of the edge. Accordingly, it is not possible to set a rain force by laser remelting for the investigated process parameters.

Outside the saturated area of the curves the following relationship applies: At same intensities larger radii are possible the smaller the line width is. This correlation also leads to the assumption that a smaller remelting depth leads to a larger radius of the remelted sheet metal edge. For this reason, the remelting depth has been determined for various process parameters that lead to different radii (fig. 9).

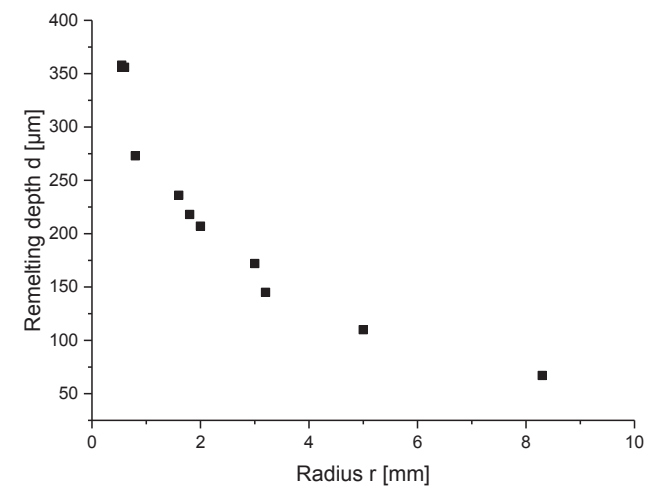

Fig. 9. Remelting depth $d$ in dependency of the radius $r$ of the laser remelted sheet metal edge for different process parameters.

Now the question arises what the maximum possible radius that can be achieved by laser remelting is. It is assumed that the main influencing factors on the rounding of the thin sheet metal edge are the surface tension and mass conservation. To achieve smaller bulges in the middle of the edge and thus a bigger radius of the rounded edge, a smaller remelting depth at the edge region is necessary due to the conservation of mass.

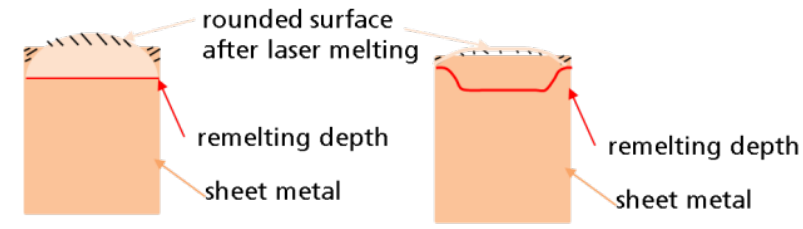

Fig. 10. Assumed schematics of the mechanisms of the bulge formation during laser remelting.

Therefore, in the next step a laser beam with reduced energy in the edge region is formed by using apertures (fig. 4, left, bottom). The results of edge rounding using the cut line are compared with those obtained with the line (fig. 10). Both laser beams have the same length with $1500 \mu \mathrm{m}$ and also the same width of $500 \mu \mathrm{m}$ in scan direction.

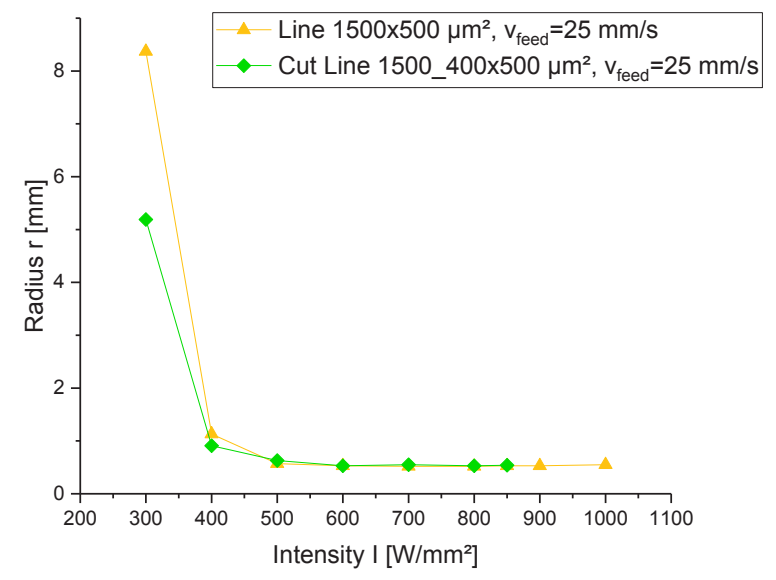

Fig. 11. Average radius $r$ of the laser remelted edge for different shaped lines and different intensities I for the feed speeds $\mathrm{v}_{\text {feed }}=25 \mathrm{~mm} / \mathrm{s}$.

It seems that the assumption of reducing the intensity in the edge regions leads to a smaller bulge, e.g. a bigger radius is not true. For small intensities the regular line shape leads to a bigger radius than the cut line.

\section{First industrial implementations}

Laser remelting of push belts for the torque transmission in CVTs lead to an increase of the fatigue strength by up to $200 \%$ in comparison to the tumbling method used previously $[2,3]$. This enables a higher torque. The pushbelt (comprising around 400 elements and 18 rings) transmits the torque between two pairs of conical pulleys. By changing the distance between the pulleys the transmission ratio can be changed continuously.

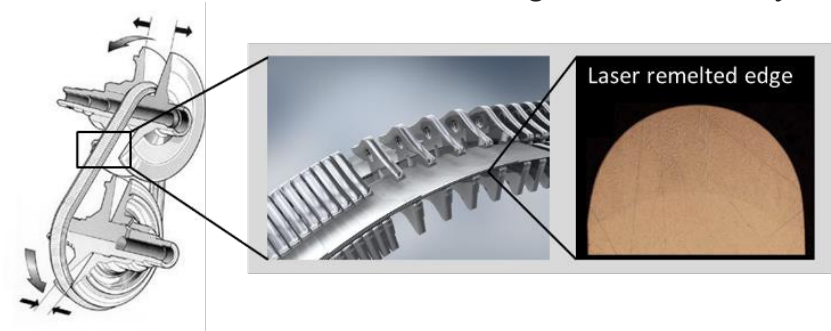

Fig. 12. Laser remelted push belts. 


\section{Summary \& Outlook}

On the austenitic stainless steel 1.4301 with an edge thickness of $1 \mathrm{~mm}$ basic correlations between process parameters and the resulting radius of the remelted sheet edge have been investigated. The most important relations are:

- Radius $\mathrm{r}$ increases with decreased laser power/intensity I and remelting depth $d$ up to the point at which the process gets unstable

- Radius $r$ increases for smaller line widths

- $\quad$ Radius $r$ increases for faster feed speed $v_{\text {feed. }}$

Above a certain intensity, which depends on the line width and the feed speed, it is not possible to further reduce the radius of the remelted edge. Regardless of the line width in feed direction, the minimum achievable radius is constant with $\mathrm{r} \approx 0.55 \mathrm{~mm}$.

The maximum radius that can be achieved is limited by the instability of the remelting process due to small intensities or remelting depths $(\mathrm{r} \approx 8 \mathrm{~mm})$. It seems that larger radii are possible the smaller the line width in feed direction. Therefore one solution to further increase the radius of the remelted edge is to decrease the line width in feed direction. For the realization of smaller line widths either a new optic with significantly reduced power losses is required, or apertures have to be used which crop the beam even more. However the smaller the line width should be, the more laser power has to be cut out in the intermediate focus. In this case a laser source with a higher maximum output power is required.

The remelting of the sheet edges shows a high reproducibility. The investigations were carried out three times. The standard deviation on the resulting radii is always between 0.01 and $0.04 \mathrm{~mm}$.

\section{References}

1. T. Schmidt-Sandte, „Laserstrahlbasierte Entgratverfahren für feinwerktechnische Anwendungen“, Dissertation, Technische Universität Braunschweig, (2003)

2. Presentation of T. Kiedrowski, BOSCH, at LaP2014 in Aachen, Germany

3. Presentation of J. Vermeer, BOSCH, at AKL14 in Aachen, Germany

4. H. Brüning, F. Vollertsen, "Energy efficiency in laser rod end melting”, LIM 2015

5. H. Brüning, F. Vollertsen, "Surface accuracy achieved by upsetting of preforms generated by laser rod end melting", MATEC Web of Conferences 21, 09006 (2015) 